\title{
PERFIL METABÓLICO DURANTE O PERIPARTO DE OVELHAS DA RAÇA SANTA INÊS COM GESTAÇÃO SIMPLES E MÚLTIPLA
}

\section{METABOLIC PROFILE DURING THE PERIPARTURIENT EWES SANTA INÊS WITH SIMPLE AND MULTIPLE PREGNANCY}

\author{
Roberta Tavares Moreira1* - ORCID: https://orcid.org/0000-0003-3928-2941 \\ Lorena Campos Assis² - ORCID: https://orcid.org/0000-0002-5346-6774 \\ Eduardo Maurício Mendes Lima² _ORCID: https://orcid.org/0000-0001-7449-8428 \\ Elias Jorge Facury Filho3 - ORCID: http://orcid.org/0000-0003-0836-2105 \\ José Renato Junqueira Borges² - ORCID: https://orcid.org/0000-0002-1338-4432 \\ ${ }^{1}$ Instituto Federal de Brasília, DF. Brasil \\ 'Universidade de Brasília, DF. Brasil \\ 3Universidade Federal de Minas Gerais, MG. Brasil \\ *Autora para correspondência - betatmoreira@yahoo.com.br
}

\section{Resumo}

O perfil metabólico é uma importante ferramenta no diagnóstico de doenças metabólicas e monitoramento nutricional de animais de produção. Este trabalho teve por objetivo avaliar o escore corporal e perfil metabólico de ovelhas da raça Santa Inês criadas em sistema de semiconfinamento, ao longo do periparto, comparando esses parâmetros entre animais com gestação simples e gestação múltipla. Cinquenta e cinco ovelhas (55), sendo 43 com gestação simples e 12 com gestação gemelar, foram submetidas a coletas de sangue e avaliação do escore corporal semanalmente, entre o fim do terceiro mês de gestação e um mês pós-parto, totalizando 10 avaliações. Observaram-se diferenças na comparação entre semanas para as variáveis: Glicose, BHB, NEFA, Triglicerídeos, Cálcio, Fósforo, Uréia e Creatinina para os grupos Gestação Simples (GS) e Gestação Gemelar (GG), além de Magnésio para o primeiro grupo $(\mathrm{p}<0,005)$. A semana do parto e as semanas subsequentes apresentaram valores estatisticamente diferentes das semanas que precederam o parto na maioria das variáveis estudadas. Não houve diferença significativa na comparação entre os grupos GS e GG para os parâmetros avaliados. Em ambos os grupos houve redução no escore corporal das fêmeas acompanhadas, com uma queda na proporção de animais com EC3 e 4 e concomitante crescimento de EC2.

Palavras-chave: Ovinos. Metabolismo. Dieta. Parto. Toxemia da Prenhez.

\begin{abstract}
The metabolic profile represents an important tool in the diagnostic of metabolic diseases and in the nutritional monitoring of production animals. The main aim of this study was to evaluate the body scores and metabolic profile of sheep of the Santa Inês breed in a system of semi- confinement, during the peri-parturient. The body scores and metabolic profile were analysed between animals with single and multiple pregnancies. Fifty-five sheep were recruited for this study, of those, 43 with single pregnancy and 12 with twin pregnancies. The animals were submitted to blood collection and analyses of body score (BS) weekly, from the end of third month of pregnancy until one month postpartum, and those were analysed 10 times during this research. About the analyses throughout weeks, the study found different values to the variables Glucoses, BHB, NEFA, Triglycerides, Calcium, Phosphorus, Urea, and Creatinine for the Single Pregnancy (SP) and Twin Pregnancies (TP) groups $(\mathrm{p}<0,005)$.
\end{abstract}


Moreover, distinct rates of Magnesium was noticed in the Single Pregnancy group $(p<0,005)$. The week of birth and subsequent weeks, showed statistically different values of those from the weeks leading up to the birth in most of the variables studied. There was no significant difference in the comparison between the SP and TP groups for the parameters evaluated. In both groups, there were a decrease in the female body score, with a reduction in the proportion of animal with BS3 and 4 associated, and concomitant increase of animals with BS2 proportion.

Keywords: Sheep. Metabolism. Diet. Parturition. Pregnancy Toxaemia.

Recebido em: 26 de fevereiro de 2015

Aceito em: 17 de maio de 2019.

\section{Introdução}

A ovinocultura de corte vem ocupando um espaço cada vez mais expressivo na pecuária brasileira ao longo dos últimos anos, tendo em vista o aumento do consumo pelo mercado nacional. Segundo Ávila et al. $(2013)^{(1)}$, o consumo interno de carne ovina saltou de 70 mil para mais de 120 mil toneladas entre 2003 e 2006, volume superior ao produzido pela ovinocultura nacional, estimulando e alavancando a produção da espécie na agropecuária brasileira.

Mesmo com o grande potencial de crescimento, uma importante barreira à expansão da ovinocultura são as doenças metabólicas que acometem principalmente ovelhas em período produtivo, doenças que são, geralmente, diagnosticadas e tratadas tardiamente, culminando em perdas e prejuízos aos sistemas de produção. As doenças metabólicas são caracterizadas por um desequilíbrio entre o ingresso e o metabolismo de nutrientes (glicídios, proteínas, minerais e água) no organismo animal, onde o aporte de alimentos não é suficiente para atender à demanda nutricional de manutenção e produção( ${ }^{(2)}$.

A toxemia da prenhez é a doença metabólica de maior relevância econômica que acomete os ovinos. Ocorre durante as últimas seis semanas de gestação, quando o(s) feto(s) alcança(m) cerca de 70\% de seu crescimento ${ }^{(3)}$. É considerada como uma das mais importantes doenças causadoras de morte em ovelhas gestantes pela difícil resposta ao tratamento, onde grande parte das fêmeas afetadas vão a óbito, caracterizando alta letalidade ${ }^{(4,5)}$. Considerando-se a importância clínica e econômica da Toxemia da Prenhez, o diagnóstico precoce da doença é primordial para o sucesso do tratamento.

A enfermidade caracteriza-se por um quadro de hipoglicemia associado à acidose metabólica, cetose, esteatose hepática, sintomas neurológicos e digestivos, quadro clínico que, quando não tratado ou tratado tardiamente, leva a fêmea acometida à morte ${ }^{(6)}$. Fêmeas com gestações múltiplas muito magras ou muito gordas são mais propensas a desenvolver a toxemia da prenhez ${ }^{(7)}$. Quanto maior o número de fetos em gestação e quanto mais próximo ao parto, maiores são as demandas energéticas para manutenção materna e fetal e, consequentemente, maiores são as chances de ocorrerem desordens metabólicas se comparando às fêmeas gestantes de fetos únicos, sendo o terço final da gestação o período mais crítico para essa doença ${ }^{(8)}$.

Além da toxemia da gestação, doenças causadas por carências minerais, como hipocalcemia, também merecem atenção durante o periparto em ovinos. A hipocalcemia em ovelhas ocorre nas formas clínica e subclínica, nas últimas semanas de gestação até duas semanas pós-parto, durante o processo de mineralização do esqueleto do feto/recém-nascido. Apesar da baixa incidência (em torno de 5\%), geralmente coexiste com quadros de toxemia da gestação. A hipomagnesemia, outro transtorno 
metabólico mineral, apesar de rara na espécie ovina, está atrelada, principalmente, à dieta, tendo em vista a baixa capacidade de mobilização de reserva perante as necessidades ${ }^{(9)}$ do animal.

A avaliação do perfil metabólico tem sido primordial para o diagnóstico precoce de moléstias metabólicas nos animais de produção, uma vez que permite o monitoramento das vias metabólicas de energia, proteínas e minerais, e da higidez de órgãos vitais à produção, como o fígado, servindo como indicador do balanço metabólico ${ }^{(2,10)}$. Dessa forma, o conhecimento dos parâmetros relativos ao perfil metabólico durante o período gestacional de pequenos ruminantes torna-se uma base segura e de fácil execução ao veterinário clínico para o diagnóstico e tratamento precoces da toxemia da prenhez, evitando grandes prejuízos nos sistemas de produção.

Considerando a escassez de literaturas que abordam o perfil metabólico de ovelhas gestantes da raça Santa Inês, e dada a importância do conhecimento desses fatores para o bom manejo nutricional em um período tão crítico da produção, este trabalho teve por objetivo avaliar o escore corporal e perfil metabólico de ovelhas da raça Santa Inês criadas em sistemas de semiconfinamento, ao longo do periparto, comparando os parâmetros entre animais com gestação simples e gestação múltipla.

\section{Material e métodos}

Este trabalho foi aprovado pela Comissão de Ética no Uso Animal (CEUA) do Instituto de Ciências Biológicas da Universidade de Brasília (UnB), sob o protocolo n ${ }^{0}$ 55245/2013.

Foram utilizadas 55 ovelhas adultas, multíparas, da raça Santa Inês, provenientes de um sistema de produção semi-intensivo de ovinos localizado na região do PAD-DF, divisa entre o Distrito Federal e o estado de Goiás. A fazenda, composta por um rebanho de 900 ovinos, mantinha os animais da raça Santa Inês em sistema de semiconfinamento, manejados a pasto no período das águas (sistema agrofloresta Eucalipto/Brachiaria decumbens) para fêmeas não prenhes sem borrego ao pé e fêmeas gestantes, e piquetes de Tyfton 85 para fêmeas paridas com borrego ao pé, e suplementados com silagem de milho e concentrado durante o período da seca. $\mathrm{O}$ sal mineral era disposto à vontade aos animais. Não havia distinção de dieta por categoria ou faixa etária, sendo que todos os animais do rebanho eram submetidos às mesmas bases alimentares. Durante o período da seca, em que se passou a fase experimental de campo deste trabalho, os animais recebiam silagem misturada ao concentrado duas vezes ao dia, às 7 e 16 horas (composição da dieta durante a fase experimental - Tabela 1).

Tabela 1. Composição bromatológica da dieta oferecida diariamente por animal, no período da seca, tendo silagem de milho como base volumosa, ração produzida na propriedade como base do concentrado ( $51 \%$ fubá de milho, $25 \%$ farelo de soja, $20 \%$ farelo de trigo e $4 \%$ núcleo comercial Guabi $\otimes)$ e sal Ovinofos Turtuga $\otimes$ como fonte mineral:

\begin{tabular}{lllccc}
\hline Fonte alimentar & MS (Kg) & NDT (Kg) & PB (g) & Ca (g) & P (g) \\
\hline Silagem de milho & 1.91 & 1.15 & 150 & 6 & 4 \\
Ração & 0.09 & 0.06 & 166 & 1 & 0.6 \\
Sal mineral & 0.02 & 0 & 0 & 2.4 & 1.7 \\
Total & $\mathbf{2 . 0 2}$ & $\mathbf{1 . 2 1}$ & $\mathbf{3 1 6}$ & $\mathbf{9 . 4}$ & $\mathbf{6 . 3}$ \\
\hline
\end{tabular}

Um mês após o término da estação de monta, que se deu entre abril e junho de 2013, os lotes de ovelhas em estação reprodutiva foram submetidos à avaliação ultrassonográfica, a partir da técnica transparietal, para diagnóstico gestacional. As fêmeas adultas com prenhez confirmada aptas a 
comporem o grupo experimental foram selecionadas aleatoriamente até atingirem o número de animais necessário para a pesquisa.

A partir do terceiro mês de gestação, tempo estimado pelo período de estação de monta, as ovelhas selecionadas passaram a ser submetidas a coletas semanais de sangue em tubos Vacutainer® sem anticoagulante, por venopunção da jugular, para obtenção de soro necessário às análises bioquímicas, no volume de $20 \mathrm{ml}$ por animal. Também foram submetidas à avaliação de escore corporal (EC), por meio de inspeção geral e palpação dos processos dorsal e transverso das vértebras lombares e fossa ísquio-caudal, classificados segundo McManus et al. ${ }^{(11)}$.

O período total de coletas e avaliação de escore corporal perdurou da quinta semana pré-parto até a quarta semana pós-parto, num total de 10 avaliações por animal (Tabela 2), entre os meses de julho e novembro, coincidente com parte da estação de nascimento na propriedade (setembro a novembro).

Tabela 2. Tempos e intervalos considerados para a determinação do perfil metabólico de ovelhas da raça Santa Inês no Periparto

\begin{tabular}{ll} 
Tempo & Intervalo \\
\hline Pr 5 & 5 semanas pré-parto \\
Pr 4 & 4 semanas pré-parto \\
$\operatorname{Pr} 3$ & 3semanas pré-parto \\
Pr 2 & 2 semanas pré-parto \\
Pr 1 & 1 semana pré-parto \\
P 0 & Semana do parto \\
Po 1 & 1 semana pós-parto \\
Po 2 & 2 semanas pós-parto \\
Po 3 & 3 semanas pós-parto \\
Po 4 & 4 semanas pós-parto
\end{tabular}

Não foi possível identificar a existência de gestação simples ou gemelar no momento do exame ultrassonográfico, sendo que alguns animais já estavam em fase gestacional superior a um mês, momento onde era impossível ter certeza do número de fetos gestantes. Logo, a separação dos grupos experimentais Gestação Simples (GS) e Gestação Gemelar (GG) só se deu no momento do parto. Dessa forma, os grupos Gestação Simples e Gestação Gemelar foram compostos por 43 e 12 ovelhas, respectivamente.

Imediatamente após a coleta de sangue, utilizou-se parte das amostras para avaliação, in loco, dos níveis de Glicose (GLI) e Beta-hidroxibutirato (BHB), por intermédio do medidor portátil Optimum Xceed ${ }^{\circledR}$. Os tubos contendo as amostras de sangue, previamente identificados, foram acondicionados em caixas isotérmicas com gelo até o fim das coletas e posterior transporte.

No Laboratório de Anatomia Veterinária da FAV/UNB, as amostras eram centrifugadas (centrífuga Centribio $\left.{ }^{\circledR}\right)$, no mesmo dia das coletas, a $3000 \mathrm{rpm}$ por 10 minutos para extração de soro, o qual era aliquotado em tubos Eppendorf® de $2 \mathrm{ml}$ (no mínimo 4 alíquotas por animal por coleta) e mantido congelado a $-18^{\circ} \mathrm{C}$ até o momento da realização das demais análises bioquímicas, que foram feitas ao fim de toda a sequência de coletas.

As analises bioquímicas, que incluíram a dosagem de Proteínas Totais (PROT), Albumina (ALB), Cálcio (Ca), Fósforo (P), Magnésio (Mg), Ureia (UR), Creatinina (CRT), e a medição da atividade das enzimas Aspartatoaminotranferase (AST) e Gama glutamiltransferase (GGT), foram realizadas no Laboratório de Anatomia Veterinária da FAV-UNB, a partir de Kits comerciais Labtest ${ }^{\circledR}$, em 
espectrofotômetro colorimétrico semi-automático (BioPlus BIO 2000®). A dosagem de Globulinas (GLB) foi realizada com base na diferença dos valores de dosagem de proteínas totais e albumina. A dosagem de Ácidos Graxos não Esterificados (NEFA) e Triglicerídeos (TRIG) foi realizada no Laboratório de Patologia Clínica da Escola de Veterinária da UFMG, em espectrofotômetro automático (COBAS Mira Plus ${ }^{\circledR}$ ) com suporte em kits comerciais Randox ${ }^{\circledR}$ e Kovalent ${ }^{\circledR}$, respectivamente.

A partir dos resultados obtidos na dosagem de BHB, realizou-se levantamento de possíveis quadros subclínicos de Cetose, considerando-se valores entre 0.8 e $3 \mathrm{mmol} / \mathrm{L}$ da variável como indicativo da

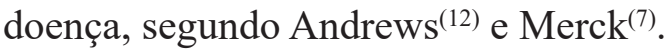

Os resultados obtidos no estudo foram expressos com fundamento na análise descritiva através de média e desvio padrão da média, em um delineamento inteiramente casualizado com parcelas subdivididas, sendo os tratamentos (GS e GG) as parcelas, e os tempos as subparcelas.

Os dados obtidos através das avaliações de perfil bioquímico foram analisados pelo programa Graphpad Prism 6.01®, por análise de variância seguida do teste de Holm Sidak's, para dados com distribuição Normal, e Teste de Friedman, para dados que não seguiram o padrão Normal, traçandose comparações múltiplas nas diversas variáveis analisadas entre semanas para o mesmo grupo. Na comparação entre o grupo Gestação Simples e Gestação Gemelar utilizou-se o Teste t Pareado, para parâmetros com distribuição Normal e Teste de Wilcoxon para valores sem distribuição Normal. Em todos os casos, considerou-se $p \leq 0,05$ como estatisticamente significativo.

\section{Resultados}

Os valores médios e Desvio Padrão da Média (DP) para as variáveis estudadas na avaliação do perfil metabólico de ovelhas da raça Santa Inês com gestação simples e gemelar (nos tempos Pr5, Pr4, Pr3, Pr2, Pr1, P0, Po1, Po2, Po3 e Po4) encontram-se nas Tabelas 3 e 4, respectivamente.

A partir da análise temporal do grupo GS, durante o periparto, foram observadas diferenças estatísticas nas comparações entre semanas para variáveis Glicose, BHB, NEFA, Triglicerídeos, Cálcio, Fósforo, Magnésio, Ureia e Creatinina (evidenciado por letras diferentes na mesma linha correspondente da tabela 3). Na análise temporal do grupo GG as diferenças foram observadas nas variáveis Glicose, BHB, NEFA, Triglicerídeos, Cálcio, Fósforo, Ureia e Creatinina (tabela 4).

Ao avaliar o perfil metabólico energético, os níveis de glicose das ovelhas do grupo GS apresentaramse mais elevados $(\mathrm{p}<0,05)$ em todo o período pós-parto se comparado ao pré-parto. O aumento da glicemia iniciou-se na semana anterior ao parto ( $\operatorname{Pr} 1)$, mantendo-se estável a partir da semana do parto (P0). Comportamento semelhante foi observado no grupo GG, com níveis de glicose mais altos em todas as semanas pós-parto quando comparadas às semanas correspondentes ao fim da gestação $(\mathrm{p}<0,05)$, e elevação dessa variável a partir da semana do parto. $\mathrm{Na}$ análise comparativa entre os grupos GS e GG não foram observadas diferenças estatisticamente significativas.

$\mathrm{Na}$ avaliação da variável BHB, para o grupo GS, observou-se elevação nos níveis médios a partir da semana Pr1, com valores mais altos da variável em todas as semanas seguintes se comparados às quatro primeiras semanas avaliadas $(\mathrm{p}<0,05)$. Em GG, níveis mais altos de BHB foram encontrados a partir da semana do parto, observando-se diferença nos valores médios entre as semanas pré e pósparto $(p<0,05)$. Na comparação entre GS e GG, também não se observou diferença entre os grupos. Durante as dez semanas avaliadas, $37,2 \%$ das ovelhas com gestação simples desenvolveram cetose subclínica, enquanto que no grupo GG, a proporção de animais com o quadro foi de $66,6 \%$. 


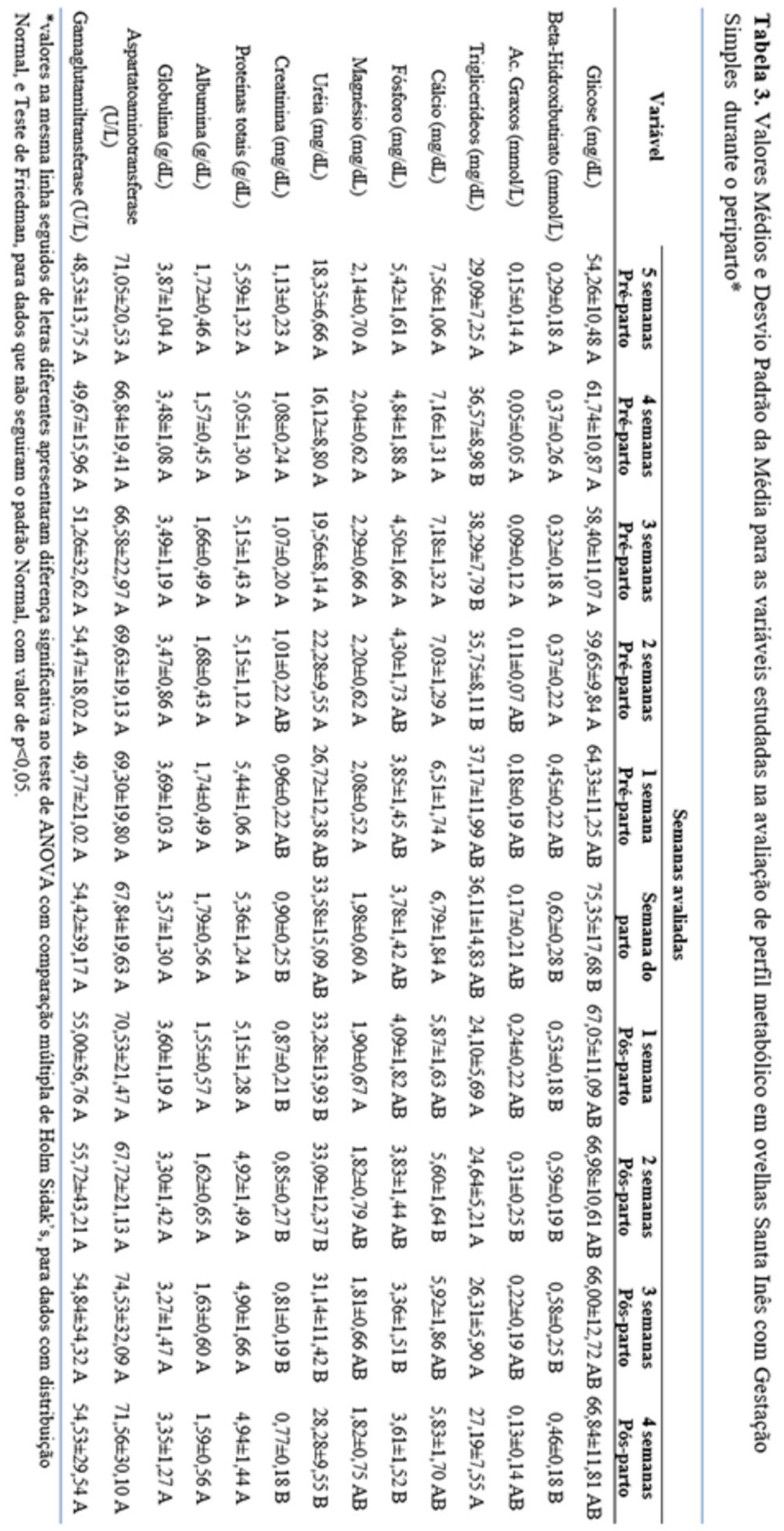




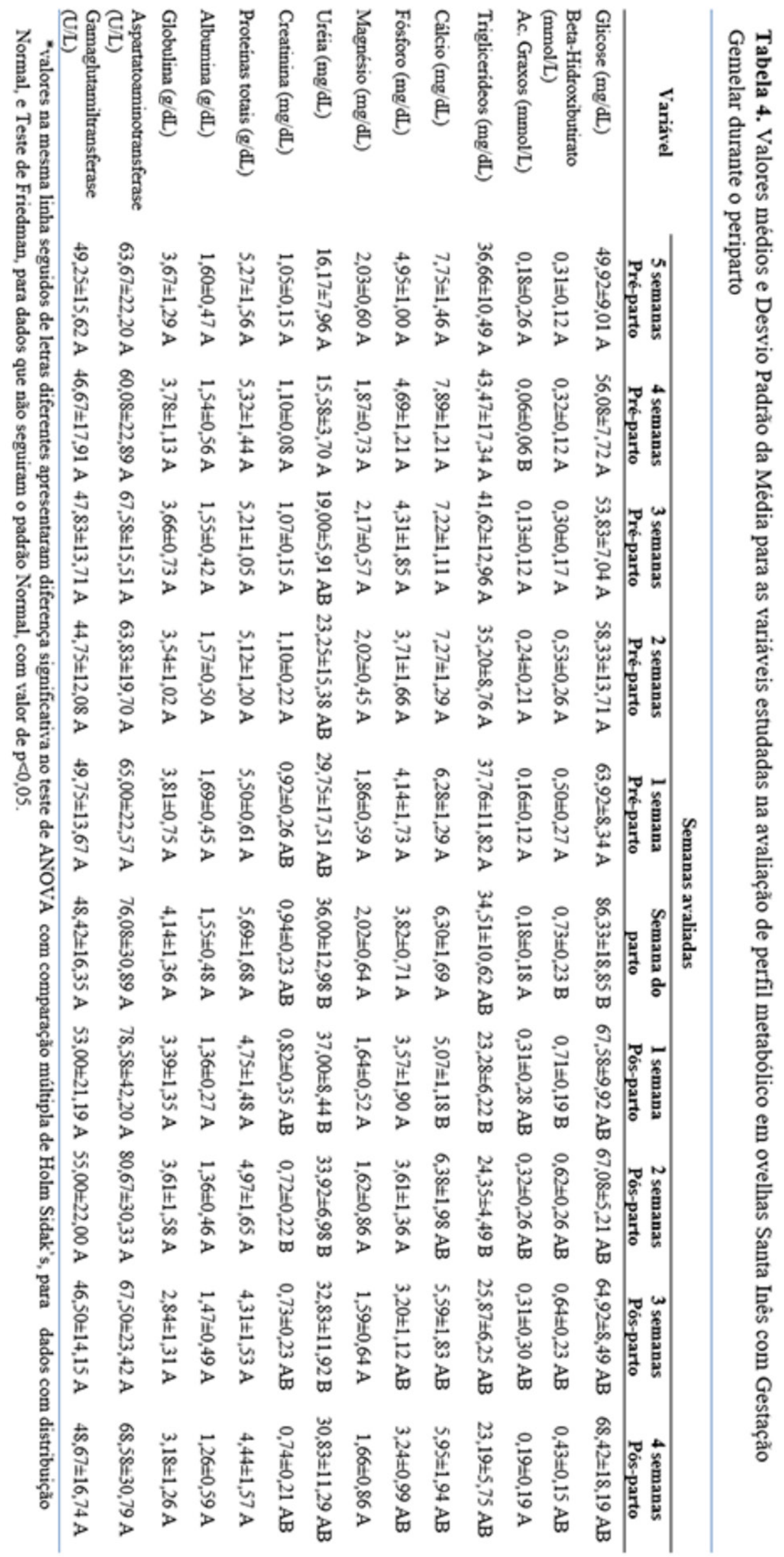


$\mathrm{Na}$ avaliação dos ácidos graxos não esterificados (NEFA), observou-se elevação nos níveis médios da variável, tanto em GS como em GG, a partir de Pr2 até Po2, onde as semanas pós-parto Po1 a Po3 apresentaram os maiores valores observados $(p<0,05)$. Na comparação entre GS e GG, as semanas $\operatorname{Pr} 3$ e Pr2 foram estatisticamente diferentes $(p=0,01)$, onde os valores médios da variável para GG foram superiores.

Os níveis médios de triglicerídeos apresentaram-se mais elevados nas semanas pré-parto, se comparados aos valores observados nas semanas pós-parto $(\mathrm{p}<0,05)$. Na comparação de GS e GG, a semana Po4 mostrou-se com níveis médios mais baixos da variável em GG $(\mathrm{p}=0,006)$.

Quanto ao perfil metabólico mineral, na avaliação dos níveis de Cálcio, observou-se, tanto em GS como em GG, valores inferiores da variável nas semanas Po1 a Po4 se comparadas às semanas anteriores $(p<0,05)$. Na comparação entre os grupos GS e GG, observou-se diferenças na semana do parto $(\mathrm{p}=0,002)$, com o grupo gestação gemelar com níveis inferiores. Em se tratando da variável Fósforo, no grupo GG observou-se níveis decrescentes da variável ao longo das semanas $(\mathrm{p}<0,05)$. No grupo GG, apenas as duas últimas semanas avaliadas foram inferiores às anteriores $(\mathrm{p}<0,05)$. Nenhuma diferença foi observada no comportamento da variável Fósforo ao longo das semanas entre GS e GG. Na avaliação do mineral Magnésio, as três últimas semanas avaliadas (Po2 a Po4) foram estatisticamente inferiores à semana $\operatorname{Pr} 3(\mathrm{p}<0,05)$. Não houve diferença estatística na comparação entre semanas avaliadas em GG. Na comparação entre os dois grupos pesquisados, também não se observou diferença ao longo das semanas.

Quanto ao comportamento do perfil metabólico proteico, em se tratando dos níveis de Ureia, as semanas Pr1 a Po4 apresentaram valores superiores às semanas anteriores $(p<0,05)$ no grupo GS, evidenciando uma elevação nos valores médios da variável ao longo do período avaliado. No grupo GG, a elevação dos níveis da variável se deu a partir da semana Pr3, onde o período pós-parto apresentou-se com valores estatisticamente superiores ao período pré-parto $(p<0,05)$. Ao se comparar os grupos GS e GG, nenhuma diferença estatística foi notada entre as semanas avaliadas.

Com relação aos níveis de Creatinina, no grupo GS as duas primeiras semanas avaliadas foram estatisticamente diferentes das demais semanas, evidenciando redução dos níveis médios da variável ao longo das semanas $(\mathrm{p}<0,05)$. Essa diminuição dos níveis séricos médios foi observada a partir da semana Pr3 no grupo GG $(\mathrm{p}<0,05)$. Não houve diferença estatística na comparação entre os grupos.

Na avaliação das variáveis Proteínas Totais, Albumina e Globulina, não foram observadas diferenças na comparação entre semanas, tanto para o grupo GS como GG. Semelhantemente, na comparação entre os grupos GS e GG, não foi constatada diferença entre as semanas avaliadas para as variáveis.

$\mathrm{Na}$ avaliação do perfil hepático, não se observaram diferenças entre semanas, tanto para os grupos GS e GG, nas variáveis AST e GGT. Na comparação entre grupos, não foram observadas diferenças.

$\mathrm{Na}$ avaliação do comportamento do Escore Corporal em ambos os grupos, Gestação Simples e Gestação Gemelar, observou-se no primeiro grupo uma elevação na proporção de animais com EC2 concomitante à redução na porcentagem de animais com EC3 e EC4 (Tabela 5), sendo o EC3 o predominante na última semana avaliada. A análise de escore corporal para o grupo GG demonstrouse bem mais variável que em GS. Semelhantemente ao grupo GS, houve aumento da proporção de animais com EC2 e redução da proporção de animais com EC3 e 4, porém em GG, a redução do EC proporcionou a inversão na disposição destes, sendo evidenciado o EC2 como predominante na última semana avaliada (Tabela 6). 
Tabela 5 - Frequência de Escore Corporal no grupo Gestação Simples

\begin{tabular}{|c|c|c|c|c|c|c|c|c|c|c|}
\hline \multirow[b]{2}{*}{$\begin{array}{l}\text { Escore } \\
\text { Corporal }\end{array}$} & \multicolumn{10}{|c|}{$\begin{array}{l}\text { Distribuição (em \%) de animais com respectivos valores de EC ao longo das semanas } \\
\text { avaliadas - Grupo GS }\end{array}$} \\
\hline & $\begin{array}{c}5 \\
\text { semanas } \\
\text { Pré- } \\
\text { parto } \\
\end{array}$ & $\begin{array}{c}4 \\
\text { semanas } \\
\text { Pré- } \\
\text { parto }\end{array}$ & $\begin{array}{c}3 \\
\text { semanas } \\
\text { Pré- } \\
\text { parto }\end{array}$ & $\begin{array}{c}2 \\
\text { semanas } \\
\text { Pré- } \\
\text { parto }\end{array}$ & $\begin{array}{c}\text { I } \\
\text { semana } \\
\text { Pré- } \\
\text { parto }\end{array}$ & $\begin{array}{l}\text { Semana } \\
\text { do parto }\end{array}$ & $\begin{array}{c}1 \\
\text { semana } \\
\text { Pós- } \\
\text { parto }\end{array}$ & $\begin{array}{l}2 \\
\text { semanas } \\
\text { Pós- } \\
\text { parto }\end{array}$ & $\begin{array}{c}3 \\
\text { semanas } \\
\text { Pós- } \\
\text { parto }\end{array}$ & $\begin{array}{c}4 \\
\text { semanas } \\
\text { Pós- } \\
\text { parto }\end{array}$ \\
\hline 1 & 0,00 & 0,00 & 0,00 & 0,00 & 0,00 & 0,00 & 0,00 & 0,00 & 0,00 & 0,00 \\
\hline 2 & 11,63 & 16,28 & 25,58 & 27,91 & 25,58 & 32,56 & 30,23 & 37,21 & 39,53 & 39,53 \\
\hline 3 & 81,40 & 76,74 & 65,12 & 65,12 & 69,77 & 62,79 & 65,12 & 60,47 & 60,47 & 60,47 \\
\hline 4 & 6,98 & 6,98 & 9,30 & 6,98 & 4,65 & 4,65 & 4,65 & 2,33 & 0,00 & 0,00 \\
\hline 5 & 0,00 & 0,00 & 0,00 & 0,00 & 0,00 & 0,00 & 0,00 & 0,00 & 0,00 & 0,00 \\
\hline
\end{tabular}

Tabela 6 - Frequência de Escore Corporal no grupo Gestação Gemelar

\begin{tabular}{|c|c|c|c|c|c|c|c|c|c|c|}
\hline \multirow{3}{*}{$\begin{array}{l}\text { Escore } \\
\text { Corporal }\end{array}$} & \multicolumn{10}{|c|}{$\begin{array}{l}\text { Distribuição (em \%) de animais com respectivos valores de EC ao longo das semanas } \\
\text { avaliadas - Grupo GG }\end{array}$} \\
\hline & 5 & 4 & \multirow{2}{*}{$\begin{array}{c}3 \\
\text { semanas } \\
\text { Pré- } \\
\text { parto }\end{array}$} & \multirow{2}{*}{$\begin{array}{c}2 \\
\text { semanas } \\
\text { Pré- } \\
\text { parto }\end{array}$} & \multirow{2}{*}{$\begin{array}{c}1 \\
\text { semana } \\
\text { Pré- } \\
\text { parto }\end{array}$} & \multirow[b]{2}{*}{$\begin{array}{l}\text { Semana } \\
\text { do parto }\end{array}$} & \multirow{2}{*}{$\begin{array}{c}1 \\
\text { semana } \\
\text { Pós- } \\
\text { parto }\end{array}$} & \multirow{2}{*}{$\begin{array}{c}2 \\
\text { semanas } \\
\text { Pós- } \\
\text { parto }\end{array}$} & \multirow{2}{*}{$\begin{array}{c}3 \\
\text { s semanas } \\
\text { Pós- } \\
\text { parto }\end{array}$} & \multirow[b]{2}{*}{$\begin{array}{c}4 \\
\text { semanas } \\
\text { Pós- } \\
\text { parto }\end{array}$} \\
\hline & $\begin{array}{c}\text { semanas } \\
\text { Pré- } \\
\text { parto }\end{array}$ & $\begin{array}{c}\text { semana } \\
\text { Pré- } \\
\text { parto }\end{array}$ & & & & & & & & \\
\hline 1 & 0,00 & 0,00 & 0,00 & 0,00 & 0,00 & 0,00 & 0,00 & 0,00 & 0,00 & 0,00 \\
\hline 2 & 8,33 & 8,33 & 0,00 & 16,67 & 33,33 & 66,67 & 50,00 & 50,00 & 1,67 & 66,67 \\
\hline 3 & 83,33 & 83,33 & 91,67 & 75,00 & 58,33 & 33,33 & 50,00 & 50,00 & 8,33 & 33,33 \\
\hline 4 & 8,33 & 8,33 & 8,33 & 8,33 & 8,33 & 0,00 & 0,00 & 0,00 & ,00 & 0,00 \\
\hline 5 & 0,00 & 0,00 & 0,00 & 0,00 & 0,00 & 0,00 & 0,00 & 0,00 &, 00 & 0,00 \\
\hline
\end{tabular}

\section{Discussão}

Independentemente do tipo gestacional, simples ou gemelar, o periparto é considerando um período crítico às ovelhas em fase produtiva, tendo em vista a grande demanda nutricional para o crescimento fetal (correspondente a 70-80\% nas últimas seis semanas de gestação), parto, produção de leite e mantença ${ }^{(13)}$. Qualquer desequilíbrio nesse momento, que ultrapasse a capacidade de adaptação do animal, pode ser fator determinante ao desenvolvimento de doenças metabólicas, muitas vezes irreversíveis. Os resultados encontrados evidenciaram a grande variabilidade no comportamento dos parâmetros relacionados ao perfil metabólico avaliados, demonstrando a capacidade de adaptação fisiológica das ovelhas às demandas em cada momento do periparto, para garantir condições de homeostase.

$\mathrm{Na}$ avaliação do perfil metabólico energético, observou-se, tanto no grupo GS quanto em GG, uma elevação nos níveis de glicose até a semana correspondente ao parto (P0). Valores crescentes nos níveis de glicose até o parto também foram observados por Oliveira et al. ${ }^{(8)}$. Semelhantemente, Cardoso et 
al. ${ }^{(14)}$, encontraram pico de glicemia correspondente à semana do parto. Em ambos os grupos, GS e GG, os níveis de glicose mantiveram-se constantes após o parto e dentro dos padrões de referência da espécie ${ }^{(15)}$ em todo o período avaliado, evidenciando um eficiente mecanismo regulatório das concentrações sanguíneas de glicose. Acredita-se que a elevação dos níveis da variável na semana do parto, se comparada às semanas precedentes, esteja relacionada ao aumento nos níveis de cortisol em decorrência do estresse do parto, hormônio estimulador da gliconeogênese, tornando a glicose mais biodisponível ${ }^{(16)}$.

Ao se avaliar o comportamento da variável BHB, todos os valores médios encontrados ao longo dos períodos avaliados, em ambos os grupos, estiveram dentro dos valores de referência para a espécie $^{(7)}$, demonstrando a manutenção das condições de homeostase. Mesmo em conformidade com os parâmetros de referência, o aumento nos níveis médios até a semana do parto e a manutenção de valores médios relativamente mais altos ao longo das semanas seguintes, para os dois grupos, evidenciaram a mobilização de tecidos de reserva como mecanismo compensatório às demandas energéticas. Ou seja, em um possível estado de Balanço Energético Negativo, com a ocorrência de lipólise e partição de energia, reflete-se em uma possível queda de insulina e, consequentemente, aumento de glicose sérica, como evidenciada anteriormente. Observou-se tanto em GS quanto em GG a ocorrência de quadros de cetose subclínica, quadros que reforçam os achados referentes à elevação dos níveis de BHB. Em vacas leiteiras em produção, Duffield et al. ${ }^{(17)}$, descreveram uma proporção de até $25 \%$ de animais com quadro de cetose subclínica. No presente trabalho, foram encontradas $37 \%$ e $66 \%$ de cetose subclínica nos grupos GS e GG, respectivamente, demonstrando alta ocorrência de cetose subclínica em ovelhas durante o periparto. Tal achado ratifica a importância de adotar como rotina a dosagem de BHB tanto para auxiliar na nutrição quanto no diagnóstico precoce de doenças metabólicas.

Ao se avaliar o comportamento dos níveis séricos médios de ácidos graxos não esterificados (NEFA), notou-se que os mesmos apresentaram tendência muito semelhante aos níveis de BHB, reafirmando um quadro de mobilização de tecidos de reserva durante o periparto e, portanto, de déficit energético. Os níveis de triglicerídeos também evidenciaram o quadro de deficiência energética, tendo em vista que apresentaram queda entre o pré e pós-parto, evidenciando sua utilização como fonte energética pelas ovelhas avaliadas e concomitantemente à sua queda, observou-se elevação nos níveis de BHB e NEFA.

Na avaliação do perfil metabólico proteico, observou-se que as variáveis PROT e ALB apresentaremse abaixo do padrão de referência para a espécie durante todas as semanas avaliadas, para ambos os grupos, GS e $\mathrm{GG}^{(15)}$. De acordo com Gonzales ${ }^{(18)}$, a concentração de proteínas plasmáticas tende a sofrer queda fisiológica algumas semanas antes do parto, recuperando-se logo após o mesmo. Porém, neste trabalho, os níveis dessa variável mantiveram-se baixos durante todo o período avaliado. Tendo em vista que os níveis de proteínas séricas estão intrinsecamente relacionados à nutrição, possíveis falhas na dieta fornecida aos animais podem justificar tal achado. Considerando como base o NRC ${ }^{(19)}$, a dieta fornecida aos ovinos (evidenciada na Tabela 1), não atende às exigências nutricionais de energia e proteínas das fêmeas de ambos os grupos após o parto, justificando, possivelmente, os níveis baixos de proteínas totais nesse período. Fontes alimentares pobres em proteínas e energia desencadeiam o decréscimo nos níveis de proteínas circulantes no sangue, isso porque para os ruminantes, a energia disponível na dieta é essencial à constituição da microbiota rumenal, a qual dará origem à proteína microbiana utilizada pelo animal como fonte nutricional de aminoácidos, além da própria proteína disponível no alimento oferecido.

Da mesma forma, a albumina sofre interferência direta da dieta. Considerando o período estudado neste trabalho, os baixos níveis observados das duas variáveis apresentadas anteriormente, 
possivelmente devem-se à alta demanda proteica somada a uma deficiência na dieta fornecida aos animais. Acredita-se também que a albumina sérica possa decrescer em decorrência de sobrecarga hepática, por acúmulo de lipídios no fígado, e pelo aumento da demanda de aminoácidos para síntese de proteínas lácteas ${ }^{(13)}$. Infestações helmínticas gastrointestinais também podem ser responsáveis pelos baixos níveis observados de proteínas totais e albumina, justificando o achado durante todo o período avaliado. A espoliação desencadeada por alta infestação helmíntica leva os animais parasitados a sofrerem déficit energético proteico, predispondo-os ao desenvolvimento de cetose e quadros clínicos de Toxemia da Gestação ${ }^{(12)}$. Tendo em vista que na propriedade o controle de endoparasitos era feito apenas com a utilização trimestral de anti-helmíntico, sem nenhum exame copro-parasitológico pré e pós-tratamento, não foi possível comprovar a eficácia do controle parasitário adotado.

Os níveis de globulina encontrados mantiveram-se dentro dos padrões de referência da espécie ${ }^{(15)}$. Tendo em vista a migração de imunoglobulinas para a composição do colostro, esperava-se que os níveis da variável globulina apresentassem mais baixos na semana do parto, fato que não foi observado. Baixos níveis de proteína na dieta desencadeiam queda nos níveis de albumina, sem alterações nos níveis de globulina ${ }^{(13)}$, coincidindo com os resultados encontrados.

Quanto aos resultados obtidos na avaliação da variável Ureia, mesmo dentro dos parâmetros de normalidade para a espécie ovina, observaram-se níveis crescentes até a semana do parto em ambos os grupos estudados, que se mantiveram relativamente constantes nas semanas seguintes. Níveis aumentados de ureia podem estar relacionados a deficientes concentrações energéticas na dieta, dada a dificuldade de utilização dos compostos nitrogenados para produção de proteínas pela microbiota rumenal, fazendo com que a amônia seja absorvida mais intensamente pela parede do rúmen ${ }^{(18)}$. Ao se avaliar os níveis de ureia conjuntamente ao BHB e proteínas totais, é possível afirmar que o aumento observado no primeiro parâmetro teve relação com o aumento da demanda nutricional nas fêmeas em estudo, associado a baixos níveis energético-protéicos na dieta oferecida, principalmente dada a maior exigência nutricional no pós-parto, resultando em gliconeogênese hepática através de mobilização aminoácidos musculares ${ }^{(20)}$, com concomitante queda no escore corporal.

Contrariamente ao observado na variável ureia, os níveis de Creatinina apresentaram-se decrescentes ao longo das semanas avaliadas, tanto em GS quanto em GG. Em ambos os grupos, os valores médios encontrados mantiveram-se abaixo dos padrões de referência para ovinos. Baixos níveis de creatinina são compatíveis com quadros de insuficiência hepática e alterações musculares degenerativas ${ }^{(18)}$. Tendo em vista que nenhum desses parâmetros foi observado nos animais estudados, não se encontrou na literatura uma explicação plausível para tal perfil.

Quanto ao perfil metabólico mineral, observou-se que os valores encontrados para as variáveis Ca, $\mathrm{P}$ e Mg tanto em GS quanto em GG, estiveram abaixo dos índices de referência para a espécie ${ }^{(15)}$. Com exceção dos níveis médios de magnésio em GG, as demais variáveis minerais apresentaram níveis decrescentes ao longo das semanas avaliadas, em ambos os grupos estudados. Tendo em vista que os valores utilizados como referência para o perfil mineral na espécie ovina são provenientes de estudos desenvolvidos em países com condições climáticas e em raças distintas dos dados deste trabalho, somado a achados de outros trabalhos nacionais ${ }^{(21,8)}$, os quais também encontraram níveis minerais abaixo dos valores de referência propostos, deduz-se que há necessidade de revisão nos valores de referência para minerais na espécie ovina sob condições de criação brasileiras, em especial a raça Santa Inês.

Cabe destacar também que a composição mineral da dieta oferecida aos animais em questão atenderam à exigência nutricional ${ }^{(19)}$ durante o período de pré-parto, mostrando-se insatisfatória apenas no pósparto para ambos os grupos, o que justifica a redução nos teores minerais observados ao longo das 
semanas avaliadas. Porém, mesmo em valores decrescentes, os níveis médios dos minerais mostraramse abaixo dos valores de referência ao longo de todas as semanas, reforçando o questionamento quanto aos padrões de referência propostos.

O cálcio é um mineral com alto controle endócrino, o que reflete em sua pequena variação sanguínea ${ }^{(22)}$. Valores baixos de $\mathrm{Ca}$ demonstram, portanto, a alta demanda metabólica, o que condiz com o período avaliado neste trabalho, tendo em vista a grande necessidade do mineral para composição do esqueleto fetal ao final da gestação, além da necessidade contrátil uterina no parto e produção de leite no pósparto. Quadros de hipocalcemia em ovelhas são mais frequentes no terço final da gestação até duas semanas pós-parto, período em que há grande mobilização para o esqueleto fetal e produção de leite, já que o leite ovino apresenta concentração de Ca duas vezes maior se comparado ao da espécie bovina $^{(9)}$. Na comparação entre os grupos GS e GG, somente a semana T0 apresentou diferença estatística quanto à variável $\mathrm{Ca}$. Os níveis de Ca para as fêmeas com gestação gemelar $(6,30 \mathrm{mg} / \mathrm{dL})$ foram estatisticamente inferiores se comparados às com gestação simples $(6,79 \mathrm{ml} / \mathrm{dL})$, demonstrando maior demanda pelo mineral durante a semana do parto para o primeiro grupo, possivelmente pela maior necessidade para consolidação dos esqueletos fetais ou pela maior contratilidade uterina.

O fósforo também é um mineral de grande importância na regulação metabólica. Sua regulação sanguínea é feita concomitantemente ao $\mathrm{Ca}$, porém seus níveis são mais variáveis, uma vez que tem participação direta nos metabolismos energético e protéico, além de vários sistemas enzimáticos ${ }^{(22)}$. Considerando-se os baixos níveis encontrados do referido mineral neste trabalho, é possível afirmar que a suplementação oferecida aos animais foi insuficiente à demanda dos mesmos, principalmente no pós-parto.

Semelhantemente ao Fósforo, os níveis de $\mathrm{Mg}$ circulante encontrados também apresentaramse abaixo dos padrões de referência para a espécie ovina. Considerando-se que o mineral $\mathrm{Mg}$ tem seus níveis intrinsecamente relacionados à ingestão na dieta, os baixos valores encontrados também podem ser relacionados ao desbalanço entre demanda e consumo. Em quadros em que há lipólise, decorrente da falta de energia, há uma tendência à redução nos níveis de $\mathrm{Mg}$ pelo seu consumo na mobilização de tecidos de reserva ${ }^{(13)}$. Considerando-se o período avaliado neste trabalho, em que houve mobilização de tecido de reserva evidenciado pelo aumento nos níveis de BHB ao longo das semanas, tal mobilização pode justificar os baixos níveis de $\mathrm{Mg}$ observados.

$\mathrm{Na}$ avaliação do perfil hepático, através da atividade das enzimas AST e GGT, observou-se níveis estatisticamente constantes tanto em GS como em GG, mantendo-se dentro dos valores de referência para a espécie ovina durante todo o período avaliado ${ }^{(15)}$. Tais achados evidenciam um estado de higidez hepática, visto que níveis aumentados de AST e GGT indicam quadros de lipidose e cirrose hepática, compatíveis com o desenvolvimento de toxemia da gestação ${ }^{(18,23)}$. Cal et al. ${ }^{(24)}$ observaram elevação na atividade de AST em ovelhas a partir de 130 dias de gestação com toxemia, níveis correlacionados positivamente com o grau de vacuolização no fígado, evidenciando que a enzima pode ser um bom indicador de alterações hepáticas decorrentes de quadros de toxemia da prenhez. Santos et al. ${ }^{(25)}$ também observaram aumento na atividade enzimática de AST e GGT em ovelhas diagnosticadas com quadros de toxemia da gestação, demonstrando a dosagem dessas variáveis como bons indicadores de alterações metabólicas onde há comprometimento hepático.

Com exceção da variável cálcio, as demais variáveis analisadas não demonstraram diferença na comparação entre os grupos GS e GG. Levando-se em consideração o fato de a gestação gemelar estar intimamente relacionada à Toxemia da Gestação ${ }^{(26,27,25,7)}$, fato justificado pela maior exigência nutricional na gemelaridade se comparado a gestações simples, a semelhança estatística em ambos os grupos avaliados com relação ao comportamento das variáveis metabólicas observadas neste trabalho 
demonstra a possível existência de outros fatores de maior importância para o surgimento da doença que o número de fetos gestantes. Mais estudos são, portanto, necessários para elencarem os fatores desencadeantes da toxemia da gestação, principalmente em se tratando de gestações gemelares.

Ao se avaliar os resultados encontrados quanto à variável Escore Corporal, observou-se, tanto em GS quanto em GG, uma redução no escore geral das ovelhas ao longo das semanas analisadas. A diminuição na porcentagem de animais com EC 3 e 4 deu lugar ao aumento no número de fêmeas avaliadas com o EC 2. Em virtude da maior demanda nutricional no fim da gestação (em decorrência do crescimento fetal), parto e período de lactação (por mobilização energética para o útero e glândula mamaria, respectivamente), é aceitável e justificável a redução de escore observada neste trabalho. O que é reafirmado ainda pelos os crescentes valores nos níveis de BHB e Ureia encontrados, indicando a mobilização de tecido de reserva para mantença da homeostase das fêmeas avaliadas. Tais resultados ratificam os encontrados por Cardoso et al. ${ }^{(14)}$ que também observaram uma queda no escore corporal no parto e pós-parto em ovelhas, com valores abaixo de 3 .

\section{Conclusão}

O perfil metabólico mostrou ser uma ferramenta de grande valia para o auxílio na avaliação nutricional e possível diagnóstico precoce de doenças metabólicas em ovelhas gestantes. Os níveis de Glicose, BHB, NEFA, Triglicerídeos, Cálcio, Fósforo, Ureia e Creatinina foram os que apresentaram maior variabilidade ao longo das semanas avaliadas, diferindo suas concentrações sanguíneas no terço final da gestação em relação à semana do parto e pós-parto. Os grupos Gestação Simples e Gestação Gemelar não diferiram, ao longo das semanas avaliadas, quanto aos parâmetros mensurados no perfil metabólico. Cetose subclínica foi um achado significativo em ambos os grupos avaliados, evidenciando a condição de balanço energético negativo durante tal período. Cuidados com a sanidade e nutrição são medidas de suma importância a serem adotadas em ovelhas no periparto a fim de se evitar desequilíbrios orgânicos que possam prejudicar a saúde em uma fase de vida tão significativa à produção desses animais.

\section{Referências}

1 - Ávila VS, Fruet APB, Barbieri M, Bianchini NH \& Dorr AC. O retorno da Ovinocultura ao cenário produtivo do Rio Grande do Sul. Rev. Elet. Em Gestão, Educação e Tecnologia Ambiental. 2013. Jun. 11:2419-2426

2- Wittwer F. Diagnóstico dos desequilíbrios metabólicos de energia em rebanhos bovinos. In: Gonzales F D., Barcelos JO, Ospina H \& Ribeiro LA. O. Perfil metabólico em ruminantes: seu uso em nutrição e doenças nutricionais. Porto Alegre, Brasil, Universidade Federal do Rio Grande do Sul. 2000. 108p.

3- Melo DB, Silva TR, Medeiros JM, Almeida FC, Dantas ES, Pessoa CRM \& Simoes SV. Toxemia da Prenhez em caprinos: relato de surto. Ciência Animal Brasileira. Suplemento 1. Anais do VIII Congresso Brasileiro de Buiatria. 2009. p.123-127.

4- Schild AL. Cetose. In: Riet-Correa F, Schild AL, Lemos RAA, Borges JRJ. Doenças e Ruminantes e Equideos. 3a ed. Vol.2, Santa Maria - Pallotti. 2007.

5- Radostits OM, Gay CC, Blood DC \& Hinchcliff KW. Clínica veterinária: um tratado de doenças dos bovines, 
ovinos, suínos, caprinos e equinos. 9ed. 2002. Rio de Janeiro: Guanabara Koogan, 1737p.

6- Pugh DG. Sheep \& Goat Medicine. 1st ed. W.B. Saunders Company. 2002,468p.

7- Merck Veterinary Manual. Pregnancy Toxemia in Ewes. 9ed. Merck \& Co., Inc. WhitehouseStation, NJ USA, 2005. 2712p.

8- Oliveira RPM, Marudo AHP, Lima ES \& Oliveira FF. Perfil metabólico de ovelhas Santa Inês em diferentes fases de gestação criadas em sistema semi-intensivo no estado do Amazonas. Ciência Animal Brasileira, 2014. Goiania, jan/mar, 15(1): 81-86.

9- Brozos C, Mavrogianni V S \& Fthenaskys GC. Treatment and control of peri-parturient metabolic diseases: Pregnancy toxemia, Hypocalcemia, Hypomagnesemia. Vet clin food anim. 2011., 27:105-113.

10- Ribeiro LAO, Gonzales FHD, Conceição TR, Brito MA, La Rosa VL \& Campos R. Perfil metabólico de borregas corriedale em pastagem nativa do Rio Grande do Sul. Acta Scientiae Veterinariae, 2003. 31: 167-170.

11- McManus C, Louvandini H, Dallago B, Melo CB \& Seixas L. Escore Corporal. INCT: Informação GenéticoSanitária da Pecuária Brasileira. Série Técnica: Genética. 2010. 31p. Disponível em: http://inctpecuaria.com. br/images/informacoes-tecnicas/serie tecnica escore_corporal3.pdf. Acesso em: 28 de julho de 2014.

12- Andrews A. Pregnancy toxaemia in the ewe. In Pratice. 1997. June. 19:306-314.

13- Contreras $\mathrm{P}$, Wittwer F \& Bohmwald $\mathrm{H}$. Uso dos perfis metabólicos no monitoramento nutricional de ovinos. In: Gonzales F. H. D., Barcelos J. O., Ospina H. \& Ribeiro L. A. O. Perfil metabólico em ruminantes: seu uso em nutrição e doenças nutricionais. Porto Alegre, Brasil, Universidade Federal do Rio Grande do Sul. 2000. 108p.

14- Cardoso EC, Oliveira DF, Dourado AP, Araujo CV, Ortolani ER \& Brandão FZ. Peso e condição corporal, contagem de OPG e perfil metabólico sanguíneo de ovelhas da raça Santa Inês no periparto, criadas na região da baixada litorânea do Estado do Rio de Janeiro. Revista Brasileira de Ciência Veterinária, 2010. mai/ago, 17(2):77-82.

15- Kaneko JJ, Harvey JW \& Bruss ML. Biochemistry of Domestic. Animals. 6th ed. Academic Press, San Diego. 2008. 916p.

16- Peixoto RT. Perfil metabólico e hormonal de vacas F1 Holandês - Zebu durante o período pré e pós parto e sua relação com a reprodução. Dissertação de mestrado em Zootecnia- Escola de Veterinária, Universidade Federal de Minas Gerais, Belo Horizonte, 2011. 57p.

17- Duffield TF, Lissemore KD, McBride BW \& Leslie KE. Impact of hyperketonemia in early lactation dairy cows on health and production. J. Dairy Sci. 2009. 92: 571-580.

18- Gonzales FHD. Bioquímica clínica. In: Gonzales F. H. D. \& Silva S. C. Patologia Clínica Veterinária/texto introdutório. Porto Alegre. Universidade Federal do Rio Grande do Sul. 2008. 342p.

19- National Research Council (NRC).. Nutrient requirement of sheep: 6ed. Washington: National Academy Press, 1985. 99p.

20- Lehninger AL. Princípios de Bioquímica. 2.ed. São Paulo: Sarvier, 1995. 839p.

21- Ribeiro LAO, Mattos RC, Gonzales FHD, Wald VB, Silva MA \& Rosa VL. Perfil metabólico de ovelhas Border Leicester x Texel durante a gestação e lactação. Revista Portuguesa de Ciências Veterinárias. 2004. 99(551):155-159.

22- Gonzales FHD. Indicadores sanguíneos do metabolismo mineral em ruminantes. In: Gonzales F. H. D., 
Barcelos JO, Ospina H \& Ribeiro LAO. Perfil metabólico em ruminantes: seu uso em nutrição e doenças nutricionais. Porto Alegre, Brasil, Universidade Federal do Rio Grande do Sul. 2000. 108p.

23- Toma HS, Chiacchio SB \& Monteiro CD. Aspectos clínicos, laboratoriais, necroscópicos e métodos diagnósticos da toxemia da gestação em pequenos ruminantes. Revista Científica Eletrônica de Medicina Veterinária. 2010. Janeiro. periódico semestral. Ano VIII, n14. 17p.

24- Cal L, Borteiro C, Benechi A, Rodasi E, Abreu MN \& Gonzales Montana JR. Histological changes of the liver and metabolic correlates in ewes with pregnancy toxemia. Arquivo Brasileiro de Medicina veterinária e Zootencia. 2009. 61(2):306-312.

25- Santos FCO, Mendonça CL, Silva Filho AP, Carvalho CCD, Soares PC \& Afonso JAB. Indicadores bioquímicos e hormonais de casos naturais de toxemia da prenhez em ovelhas. Pesq. Vet. Bras. 2011.Novembro, 31(11):974-980.

26- Soares FAP, Borba Neto AV, Guimarães JA, Dantas AC, Carvalho CCD, Marques AVS \& Soares PC. Metabolismo de indicadores preditivos de Toxemia da Prenhez em ovelhas Dorper no terço final da gestação, parto e pos-parto. Rev. Ciência Animal Brasileira, 2009. Suplemento 1, Anais do VIII Congresso Brasileiro de Buiatria, p. 197-203.

27- Campos AG, Afonso JAB, Santos RA, Mendonça CL \& Guimarães JA. Estudo Clínico-laboratorial da Toxemia da Prenhez em ovelhas: análise retrospectiva. Rev. Ciência Animal Brasileira, 2010. Goiânia, jul/set., 11(3):623-628. 